\title{
Author Index Vol. 68, 1994
}

Abramovici, M. 366 Ackrill,P. 265 Agarwal,S.K. 390,509 Aguilera, A. 149 Akalin,E. 396 Akcicek,F. 396 Akhtar,M. 500 Akpolat,T. 152 Alamo, C. 393 Alavi,F.K. 112 Alessi,D. 389 Alexandre, P. 184 Al-Khader, A A. 514 Allegra,V. 41 Alonso,A. 143 Al-Rasheed,A.M. 514 AlSulaiman, M.H. 514 Altieri,P. 353 Andany, MA. 143 Andre, E. 184 Andre, J.L. 184 Anpalahan,M. 180,405 Arakawa,M. 360,403 Araque, A. 393 Ardaillou,R. 301 Arima, S. 473 Arimura, Y. 380 Arnadottir, M. 57 Arzuaga, JA. 262 Asakura, H. 20 Asano,S. 380 Asano, T. 239 Asano, Y. 71 Atabay,G. 396 Atar,S. 259 Aydin,P. 391

Badalamenti, S. 140 Bailey, R.R. 378 Baldrati,L. 141 Bancale,E. 146 Bang,B.K. 397,507 Bansal,M. 366 Barrillon,D. 145 Barros, S.G.S. 515 Basci,A. 396 Basolo,B. 399 Baumelou, A. 202 Bayle,J. 145 Becker, G. 180,405 Bedani,P.L. 512 Bellavita,P. 400 Ben-Izhak,O. 259 Bérard,E. 145 Berardi,E. 141 Bergh, J.P.W. van den 505 Beyer, M.M. 77 Birch, D. 180 Boogaerts, M.A. 192 Borrás,M. 138 Borrego, F. 274 Böttger,W.M. 505 Boubenider, SA. 500 Bouza,P. 143 Briquel,M.E. 184 Buchwald,D. 408 Buemi,M. 277 Bundschu,D. 517 Burton, CJ. 287

Çaglar,§. 152 Caldarelli, R. 140 Cameron, E.C. 128 Can,U. 391 Candiano, G. 353 Caramelo,C. 148 Carrera, F. 38 Carretta,R. 270 Carrülo,R. 149 Cassey, J. 327 Cassuto-Viguier, E. 145 Cerski, C.T.S. 515 Chan,C.H.S. 294 Chang,Y.S. 397,507 Charlesworth, D. 265 Chen,W.-P. 10 Childs,J.E. 48 Chisholm, R.J. 378 Choi,EJ. 397,507 Chui,Y.L. 294 Cintin,C. 217 Coll,R. 449 Como,G. 140 Conte,A. 449 Coppo,R. 146 Correa-Rotter, R. 489 Costa, C.A.R. 515 Cotroneo, D. 277 Cruz,C. 489

Daelemans, R. 192 Danielson, B.G. 419 Dash,S.C. 390,509 Da Suva Cardoso, M. 517 Davis, W.G. 468 De Micheli, A.G. 245

Demoz,M. 389 Desmichels, D. 202 De Stefanis, D. 389 Diaz-Gonzalez, R. 393 Dietl,P. 234 Dilber,S. 396 Dimski,D.S. 275 Di Sciascio, N. 510 Docci,D. 141

Domrongkitchaipom, S. 128 Dorhout Mees, E.J. 396 Dosunmu, B. 77 Dufresne, L.R. 133

Duncan, R.C. 245 Durand,D. 375 Durand,J.-M. 142 Dussaule, J.C. 301 Düsünsel,R. 410

Dutton,G.N. 52 Dyras,P. 510

Ebira,H. 406 Elisaf,M. 169 Epple,S. 517 Epstein,M. 245 Eriksson, L.-O. 57 Escourrou, G. 375 Estirado, E. 262

Fabbretti,G. 353 Fabris,B. 270 Falcon, T.G. 143 Fasy,T.M. 25 Fernandez, M.J. 274 FernándezLucas, M. 149 Ferrier,C. 405 Ferro, M. 146 Fiorelli,G. 140 Fischetti,F. 270 Flatau,E. 259 Flores,E. 392 Formica, M. 399 Forster,H. 245 Fraile,B. 393 Franceschini, N. 515 Franiczek, R. 519 Frazao, J.M. 38 Friedman, EA. 77 Frimat,L. 286 Frithz,G. 419 Fujii,Y. 212 Fujishima, M. 347 Fujita,H. 406

Fujitsuka,N. 280 Fujiwara, H. 104 Fukagawa, M. 221 Fukiyama, K. 80 Fukuda,N. 221 Fukuhara,S. 442 Fukuroi,T. 442 Funaro,L. 399 Furuya, H. 71 Gallego,A. 32 Gallego,N. 32 Garberi, A. 353 Gardner, J. 378 Garvía,R. 148 Geberth, S. 155 Gennadiou, M. 169 Germershausen, J.I. 57 Ghiggeri, G.M. 353 Gibbons, N. 366 Gill, J.C. 433 Gilli,P. 512 Ginevri, F. 353 Glass, G.E. 48 Go,H. 372 Golato,M. 510 Goldsmith, D.J.A. 265 
Gómez-Campderá, F.J. 392 Goncalves, L.F. 515 Gonzalo,A. 32, 155,497 Götz,R. 454 Grases, F. 449 Graziani,G. 140 Gretz,N. 462 Guclu,A. 396 Gusmano, R. 353 Güven,K. 410

Haizuka,H. 20 Hamada,T. 104 Hamamoto, K. 442 Hanamoto, N. 401 Hanesse, B. 286

Hannedouche, T. 334 Haruki, S. 212 Haskard,D.O. 294 Havens, P.L. 433 Hayashi,T. 118

Hegbrant, J. 427 Heibel,F. 334 Heidbreder, E. 454 Heidenreich, S. 481 Heidland,A. 454

Hestin,D. 286 Higgins, R.M. 265 Hiki,Y. 394 Hiraoka,M. 212 Hishiki,T. 284 Hong,S. Y. 329

Horii,A. 394 Horikawa, S. 279 Hotchandani, R.K. 390, 509 Hou,C.-H. 125 Hsu,K,-L. 125

Huang, T.-P. 437 Hübel,E. 338 Hunter, J.B. 433 Hyman,E.S. 314 Hyodo,T. 153,401

Iaina, A. 413 Ienaga,K. 280 Iijima, T. 207 lino, A. 401 Ikeda, M. 118 Ikeda,Y. 406 Ino,T. 252

íordache, A. 145 Iseki,K. 80 Ishikawa,S. 394 Isidore, C. 389 Iwai,S. 104

Jacobs, C. 202 Jaffer,S. 366 Jankowski,S. 519 Jazaerli, N. 271 Jetha,N. 128 Jha, V. 256 Joffe,P.

217 Jofre,R. 392 Juarez-Nicolas, F. 489

Kakavas,I. 388 Kallivretakis, N. 388 Kalra,O·P. 256 Kaname,S. 380 Kaneko, K. 252 Kansu,T. 391 Kaplanski,G. 142 Karabatsos, A. 169 Karstrup, S. 217 Kassen,B.O. 128 Katayama,Y. 372 Kaufmann, B.G. 505 Kawaguchi, H. 239 Kawaguchi, M. 97 Kawaguchi, S. 97 Kawaguchi, Y. 118 Kawakatsu,T. 442 Kawasaki, K. 360 Kawashima, F. 97 Kessler,M. 286

Kido,H. 442 Kiefer, T. 338 Kihara,M. 406 Kilic,H. 410 Kim,S.-T. 308 Kim,S.Y. 397,507

Kim,Y.S. 397,507 Kincaid-Smith, P. 405 Kitaoka, M. 221 Kobayashi,Y. 394 Koch, K.M. 229

Kochhar,S. 256 Kodera,S. 284 Koemer,K. 517 Koide, H. 207, 284 Kokawa,T. 442 Kokubo,T. 394 Komeyama, T. 372 Koo,W.S. 397,507 Kostogianni, G. 169 Kourti,A. 169 Kramer, R. 517 Krautzig,S. 229 Kubanek,B. 517 Kubo,S. 308 Kuhlmann,U. 338 Kuramoto, T. 207 Kuroda,T. 360 Kuroiwa, A. 308 Kurokawa, K. 221 Kurusu, A. 284

Ladefoged, S.D. 217 Lai,C.K,W. 294 Lai,KN. 294 Lang,D. 481 Lang,F. 234 Lang, P. 271

Lassale, B. 142 La Torre, M. 510 Leblanc,M. 133 LeDuc,J.W. 48 Lefèvre,P. 142 Legault,L. 133 Leichter, H.E. 433 Lembke,J.M. 112 Letona, J.M.L. de 262 Li,P.KT. 294 Liani,M. 510 Lin,C.Y. 10,437 Lindberg,B.F. 427 Linde,T. 419 Ling,H. 454 Lins, R.L. 192 Llompart, I. 449 Lloveras, J.J. 375 Lonnemann, G. 229 Lopau, K. 454 López-Gómez, J.M. 392 Losman, M.J. 25 Lozano, I.R. 143 Lübbecke, F. 268 Lui,S.F. 294 Luisetto, G. 41 Lundin,A.P. 77

Maadi,A.B. 271 McOmish,F. 514 Mampaso, F. 497 Mandolfino, T. 277 Mansfield, D.C. 52 Marcén,R. 149,497 March, J.G. 449 Marescaux, J. 334 Marichal, J.-F. 334 Marin,M.G. 400 Mårtensson, L. 427 Martimbianco, L. 41 Martinet, N. 184 Martinez-Ara, J. 274 Marx,B.D. 275 Masa,C. 262 Masumori, S. 406 Mathieu,D. 271 Matsumoto, T. 221 Mattana, J. 366 Mazerolles, C. 375 Mazzucco, G. 399 Mehls,O. 63 Mengozzi, G. 41 Mettang,T. 338 Meyers, A.M. 197 Mignon, F. 301 Miguel, J.L. 274 Minoda,S. 380 Misiani,R. 400 Mittal,R. 390,509 Miyagawa, I. 153,401 Miyakawa, T. 403 Mizusawa, T. 372 Monestier, M. 25 Montoliu,J. 138 Monzú,B. 148 Morikawa, K. 212 Morin,C. 133 Mountokalakis, T. 169 Murata,N. 282 Mutter, D. 334 Nagasawa, T. 380 Nakahama, H. 411 Nakamur, T. 153 Nakamura,K. 280 Nakamura,T. 118 Nakanishi,T. 411 Nakayama, M. 473 Nakazawa, T. 252 Nanishi,F. 347 Naruse,T. 282 Navarro,J.F. 497

Nestel,A.W. 197 Netter,P. 286 Nielsen, A.L. 427 Nielsen, C.B. 273 Nishi, S. 403 Nishikawa, M. 442 Nishime,K. 80 Noguchi,Y. 239 Nomura, S. 442 Novick,KE. 25 Nyman,R. 500

Oberman,A.S. 259 Ode,T. 401 Ogata,E. 221,380 Ogawa, T. 87 Ogura,A. 239 Ohmuro,H. 207

Ohshima,K. 97 Oite,T. 360 Ok,E. 396 Okuda,S. 347 Okutani,M. 401 Oleggini, R. 353

Oliveira,C. 38 Onoyama, K. 347 Orofmo,L. 497 Ortuño,J. 32,149,497 Osawa,A. 80 Ota,K. 279

Oura,H. 280 Ozasa,H. 279 Özbakir,Ö. 410 Ozen,S. 152 Ozgen,G. 396 Ozkuyumcu, C. 152 
Paiker,J. 197 Palella, S. 277 Panadés, MJ. 138 Pani,I. 388 Papagalanis, N. 169 Papageorgiou, K. 388 Passlick-Deetjen, J. 338 Paydas,S. 151 Pedraza-Chaverri, J. 489 Pellegrino, N. 146 PerezMaestu, R. 262 Perfumo, F. 353 Perini,L. 512 Petitclerc, T. 202 Pettersson, L. 419 Phenekos,C. 169 Piccoli,G. 146,399 Pietrzyk,J. 510 Pignone,E. 389 Pilatte,Y. 25 Pires,C. 38 Pollaris,P. 192 Praga,M. 393

\section{2}

Author Index

Preuss,H.G. 385 Prompt, C.A. 515 Pumford,N. 327

Quattrocchio, G. 146 Quereda,C. 149

Radier,C. 271 Rahmouni, A. 271 Rahn,K.-H. 481 Raine,A.E.G. 159 Ramos, J. 138 Rankin,D. 514 Rao,T.K.S. 77 Riet, G.J.A. van 505 Rigal,D. 25 Rinno,H. 207 Ritz,E. 155 Rivera, M. 32,149 Roccatello, D. 146,389 Roger, V. 301 Rollin,H.B. 197 Roman, F. 262 Ronquist, G. 419 Rostaing, L. 375 Rostoker,G. 25,271 Rowe,P.A. 52 Roy,L.F. 133

Saeed,A.A. 514 Saelens,D.A. 112 Saitoh, A. 207 Sakai,O. 118 Sakatsume, M. 403 Sakhuja,V. 256 Salvadori, S. 140 Salvati,F. 510 Sammet, A. 63 Sampietro, M. 140 Sanden, I. 63 Sandhagen,B. 419 Sasaguri, Y. 104 Sato,M. 20 Sato,T. 473 Saxena,S. 390,509 Schaar,J. 454 Schäffer,J. 229

Schärer,K. 63 Schenk,U. 338 Schindler,R. 229 Schramm, L. 454 Schütterle, G. 268 Schwartz, D. 413 Segoloni, G.P. 399 Sei,C. 284 Sekizuka,K. 284 Shah,R. 366 Shaldon, S. 229 Sheth,KJ. 433 Shibata,K. 406 Shigematsu, H. 394 Shimada,H. 403 Shimazaki, S. 252 Shimizu, F. 360 Shio,H. 401 Shirato,I. 207,284 Shiratori,K. 406 Shouzu,A. 442 Shrivastava, D. 77 Siamopoulos, K. 169 Silva,J.G 38 Singhal,P.C. 366 Singlas,E. 202 Smyth, BJ. 468 Solis,C. 274 Sonikian, M. 388 Sonzogni, A. 400 Soria,J. 202 Soubeyrand, J. 142 Spinelli,F. 277 Stoltz,J.F. 184 Suc,J.M. 375 Sudo,M. 212 Sugita,M. 411 Sugryama, T. 394 Sungur, C. 152 Sutton, R.A.L. 128 Suzuki, M. 118,442 Suzuki, O. 239 Suzuki, S. 403 Swenson,D.H. 275

Tabei,K. 71 Taboada, J. 275

Takahashi, H. 252, 372 Takahashi,K. 473 Takasugi, M. 308 Takeda,M. 372 Tamaki,M. 372 Taneda, A. 207 Tanimoto, A. 104 Tapia,E. 489 Tashiro,K. 284 Taylor, H.W. 275 Tepel,M. 481 Teruel,J.L. 497 Terzidis-Trabelsi, H. 25 Tetiker,T. 151 Thuot,C. 133 Thysell,H. 57,427 Timpanelli, R. 277 Tiwari,S.C. 390,509 Tomino,Y. 207,284 Tönshoff,B. 63 Traba,M.L. 148 Trechot,P. 286 Tsai,T.-J. 125 Tsuchida, S. 212 Tsukada,Y. 282 Tsukahara, H. 212 Tsutsui, T. 372 Turgan, Ç. 152

Uchida,S. 380 Uehara,H. 80 Ueno,M. 403 Usal,A. 151 Utas,C. 410 Uttley,W.S. 327

Vallero,A. 146 Valvuena, L. 143 Van Steenhouse, J.L. 275 Vasile, A. 41 Vasile,N. 271

Venning, M.C. 265 Verma,P.P. 256 Verstappen, V.M.C. 505 Vicari,O. 400 Villari,C. 277 Viron,B. 301

Voisin,Ph. 184 Völkl,H. 234 Voss,D.M. 378 Voudiclari, S. 388

Wada,H. 97 Waldherr,R. 63 Walls, J. 287 Wang,H. 207 Washio,M. 347 Watson, A.J. 48 Watts, M.T. 408 Weber, J. 338 Weil,B. 271 White, R.H.R. 1 Wikström,B. 419 Wizemann, V. 268 Wolf,J.P. 301 Wöll,E. 234 Wong, G.Y. '25 Wong, K.C. 294

Yabuta, K. 252 Yamaguchi, K. 442 Yamaguchi, Y. 239, 284 Yamakido, M. 87 Yamamoto, Y. 239 Yanabu, M. 442 Yang,C.W. 397,507 Yang,D.H. 329 Yano,S. 282 Yasavul,Ü. 152 Yi,H. 221 Yokozawa,T. 280 Yoon,Y.S. 397,507 Yorioka,N. 87 Yoshimura, N. 473 Yoshino,Y. 401 Yücesoy, M. 410 
Zacharof,A. 169 Zachée,P. 192 Zawada, E.T. 112 Zeier,M. 155 Zidek,W. 481 Zimmermann, J. 454 Zuckerman, MJ. 408 